\title{
Topological Structure of Dense Hadronic Matter*
}

\author{
Byung-Yoon Park ${ }^{a}$, Hee-Jung Lee ${ }^{b}$, Vicente Vento $^{b}$, $^{2}$ \\ Joon-Il Kim ${ }^{c}$, Dong-Pil $\mathrm{Min}^{c}$ and Mannque Rho ${ }^{d, e}$ \\ (a) Department of Physics, Chungnam National University, Daejon 305-764, Korea \\ (E-mail: bypark@cnu.ac.kr) \\ (b) Departament de Fisica Teòrica and Institut de Física Corpuscular \\ Universitat de València and Consejo Superior de Investigaciones Científicas \\ E-46100 Burjassot (València), Spain \\ (E-mail: hjlee@phya.snu.ac.kr, Vicente.Vento@uv.es) \\ (c) Department of Physics, Seoul National University, Seoul 151-742, Korea \\ (E-mail: jikim@phya.snu.ac.kr,dpmin@phya.snu.ac.kr) \\ (d) School of Physics, Korea Institute for Advanced Study, Seoul 130-722, Korea \\ (e) Service de Physique Théorique, CEA Saclay \\ 91191 Gif-sur-Yvette, France \\ (E-mail: rho@spht.saclay.cea.fr)
}

\begin{abstract}
We present a summary of work done on dense hadronic matter, based on the Skyrme model, which provides a unified approach to high density, valid in the large $N_{c}$ limit. In our picture, dense hadronic matter is described by the classical soliton configuration with minimum energy for the given baryon number density. By incorporating the meson fluctuations on such ground state we obtain an effective Lagrangian for meson dynamics in a dense medium. Our starting point has been the Skyrme model defined in terms of pions, thereafter we have extended and improved the model by incorporating other degrees of freedom such as dilaton, kaons and vector mesons.
\end{abstract}

\section{Introduction}

At high temperature and/or density, hadrons are expected to possess properties that are very different from those at normal conditions. Understanding the properties of hadrons in such extreme conditions is currently an important issue not only in nuclear and particle physics but also in many other related fields such as astrophysics. Data from the high energy heavy ion colliders, astronomical observations on compact stars and some theoretical developments have shown that the phase diagram of hadronic matter is far richer and more interesting than initially expected. Lattice QCD calculations have been carried out successfully at high temperature, however similar calculations at high density have not yet been possible 1]. Theoretical developments have unveiled such interesting QCD phases as color superconductivity 2. Moreover effective theories can be derived for these extreme conditions, using macroscopic degrees of freedom, by matching them to QCD at a scale close to the chiral scale $\Lambda_{\chi} \sim 4 \pi f_{\pi} \sim 1 \mathrm{GeV}[\underline{3}$.

We have followed a different path to dense matter studies by using as our starting point a model Lagrangian, in the spirit of Skyrme, which describes hadronic matter and meson dynamics respecting the symmetries of QCD. The parameters of the model are fixed by meson dynamics at zero baryon number density. À la Skyrme 4, baryons arise from a soliton solution, the skyrmion, with the topological winding number describing the baryon number. In our scheme

*Talk given at the KIAS-APCTP Symposium in Astro-Hadron Physics " Compact Stars: Quest for New States of Dense Matter," November 10-14, 2003, Seoul, Korea 
dense matter is approximated by a system of skyrmions with a given baryon number density whose ground state arises as a crystal configuration [5, 6]. Starting from this ground state our approach provides insight on the intrinsic in-medium dependence of meson dynamics. We have studied (i) the in-medium properties of the mesons and (ii) the role of the other degrees of freedom besides pions in the description of matter as it becomes denser [7, 8, , 9, 10, 11, 12,

\section{Model Lagrangians}

The original Skyrme model Lagrangian 4 reads

$$
L_{\pi}=-\frac{f_{\pi}^{2}}{4} \operatorname{Tr}\left(L_{\mu} L^{\mu}\right)+\frac{1}{32 e^{2}} \operatorname{Tr}\left[L_{\mu}, L_{\nu}\right]^{2}+\frac{f_{\pi}^{2} m_{\pi}^{2}}{4} \operatorname{Tr}\left(U+U^{\dagger}-2\right),
$$

where $L_{\mu}=U^{\dagger} \partial_{\mu} U$ and $U=\exp (i \vec{\tau} \cdot \vec{\pi}) \in S U(2)$ is a nonlinear realization of the pion fields, $f_{\pi}$ the decay constant and $m_{\pi}$ the pion mass . The second term with $e$, the Skyrme parameter, was introduced to stabilize the soliton solution.

The dilaton field $\chi$ was incorporated in the model to make it consistent with the scale anomaly of QCD [13]. The Lagrangian (11) then becomes

$$
\begin{aligned}
L_{\pi \chi}= & -\frac{f_{\pi}^{2}}{4}\left(\frac{\chi}{f_{\chi}}\right)^{2} \operatorname{Tr}\left(L_{\mu} L^{\mu}\right)+\frac{1}{32 e^{2}} \operatorname{Tr}\left[L_{\mu}, L_{\nu}\right]^{2} \\
& +\frac{f_{\pi}^{2} m_{\pi}^{2}}{4}\left(\frac{\chi}{f_{\chi}}\right)^{3} \operatorname{Tr}\left(U+U^{\dagger}-2\right) \\
& +\frac{1}{2} \partial_{\mu} \chi \partial^{\mu} \chi-V(\chi),
\end{aligned}
$$

Note the different powers of $\left(\chi / f_{\chi}\right)$ in front of each term. The last line is the Lagrangian for the free dilaton field, where $V(\chi)=\left(m_{\chi}^{2} f_{\chi}^{2} / 4\right)\left(\left(\chi / f_{\chi}\right)^{4}\left(\ln \left(\chi / f_{\chi}\right)-\frac{1}{4}\right)-\frac{1}{4}\right), m_{\chi}$ is the dilaton mass and $f_{\chi}$ its decay constant.

The vector mesons, $\rho$ and $\omega$, can be incorporated into the Lagrangian as dynamical gauge bosons of a hidden local gauge symmetry which requires the doubling of the degrees of freedom as $U=\xi_{L}^{\dagger} \xi_{R}$. This Lagrangian reads 14

$$
\begin{aligned}
L_{\pi \chi \rho \omega}= & -\frac{f_{\pi}^{2}}{4}\left(\frac{\chi}{f_{\chi}}\right)^{2} \operatorname{Tr}\left(L_{\mu} L^{\mu}\right)+\frac{f_{\pi}^{2} m_{\pi}^{2}}{4}\left(\frac{\chi}{f_{\chi}}\right)^{3} \operatorname{Tr}\left(U+U^{\dagger}-2\right) \\
& -\frac{f_{\pi}^{2}}{4} a\left(\frac{\chi}{f_{\chi}}\right)^{2} \operatorname{Tr}\left[\xi_{L} \partial_{\mu} \xi_{L}^{\dagger}+\xi_{R} \partial_{\mu} \xi_{R}^{\dagger}+i(g / 2)\left(\vec{\tau} \cdot \vec{\rho}_{\mu}+\omega_{\mu}\right)\right]^{2} \\
& +\frac{3}{2} g \omega_{\mu} B^{\mu} \\
& -\frac{1}{4} \vec{\rho}_{\mu \nu} \cdot \vec{\rho}^{\mu \nu}-\frac{1}{4} \omega_{\mu \nu} \omega^{\mu \nu}+\frac{1}{2} \partial_{\mu} \chi \partial^{\mu} \chi-V(\chi),
\end{aligned}
$$

with $\vec{\rho}_{\mu \nu}=\partial_{\mu} \vec{\rho}_{\nu}-\partial_{\nu} \vec{\rho}_{\mu}+g \vec{\rho}_{\mu} \times \vec{\rho}_{\nu}, \omega_{\mu \nu}=\partial_{\mu} \omega_{\nu}-\partial_{\nu} \omega_{\mu}$, and where $B_{\mu}$ is the topological baryon number current. The quartic Skyrme term of (11) is not present, because its stabilizing role is played here by the vector mesons.

\section{In-Medium Pion Dynamics}

In order to explain our basic strategy, we start with the Skyrme model (11). Table 1 serves to generalize the discussion to other models. The vacuum solution of (11) in the $B=0$ sector is $U=1$. Fluctuations on top of this vacuum describe pion dynamics. The Lagrangian supports 

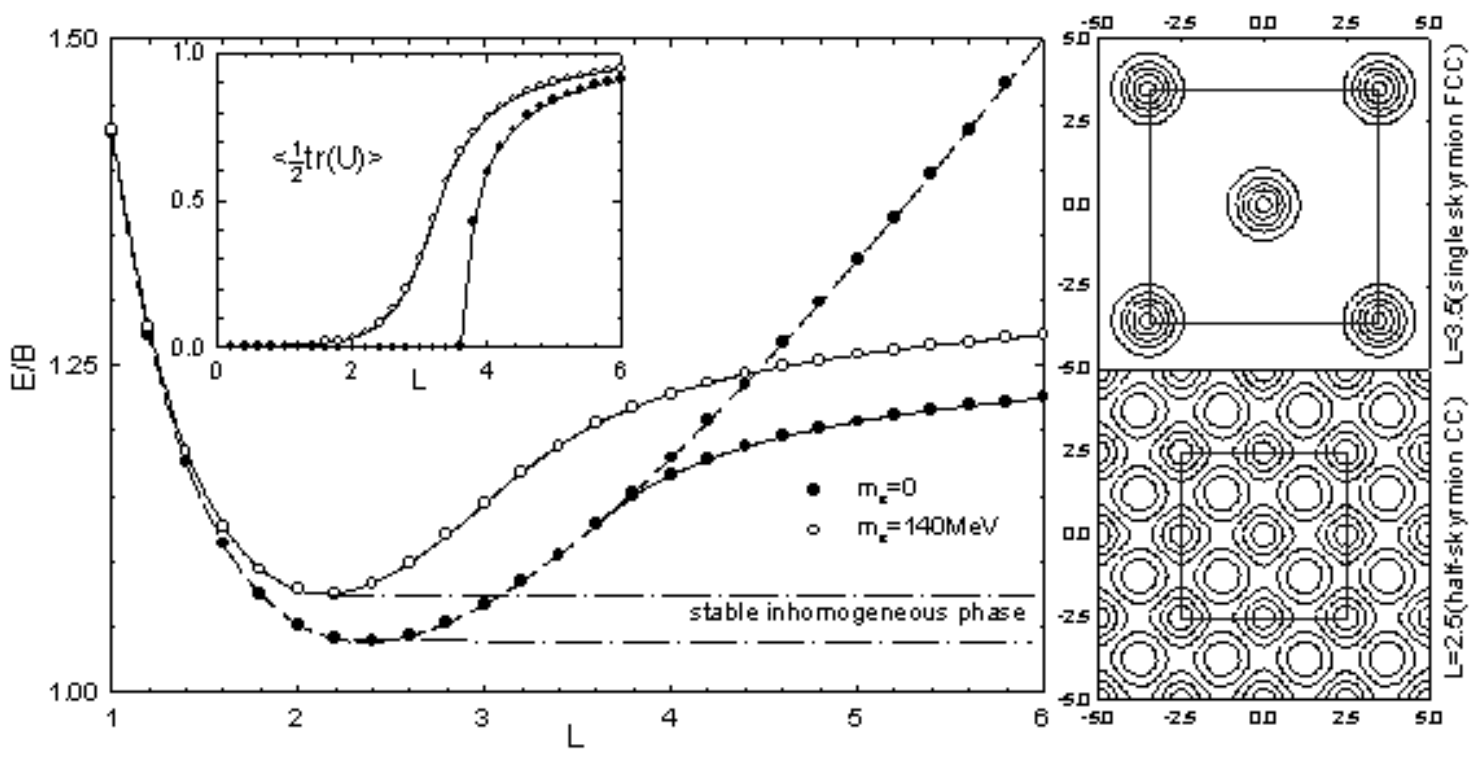

Figure 1: This figure shows the energy per skyrmion and the average value of $\sigma=\left\langle\frac{1}{2} \operatorname{Tr}(U)\right\rangle$ as a function of the FCC crystal parameter $L$ (in units of $\left(e f_{\pi}\right)^{-1}$ ). The figures in the small boxes correspond to samples of the baryon number distribution on the $x y$-plane $(z=0)$. The corresponding FCC boxes are drawn by a square.

solitons with nontrivial topological structures. The $B=1$ soliton solution with lowest energy is a "hedgehog" , $U^{B=1}=\exp (i \vec{\tau} \cdot \hat{r} F(r))$ with the boundary condition for the profile function $F(r), F(0)=\pi$ and $F(\infty)=0$. The two-skyrmion system has lowest energy when one on the skyrmions is rotated relatively to the other in isospin space, by an angle $\pi$, about an axis perpendicular to the line joining their centers. Therefore, skyrmion matter has the lowest energy in an FCC (face centered cubic) single skyrmion crystal structure, where the skyrmions at the nearest site are relatively oriented in such a low energy configuration and $U(\vec{r})$ has the symmetry structure described in Table 1.

For a given baryon number density, the lowest energy configuration can be found numerically by varying the field values at points of a discrete mesh [5] or by adjusting the coefficients of the Fourier series expansion for the fields with specific symmetries 6 .

We show in Figure 1 the energy per baryon $E / B$ of skyrmion matter and $\sigma$, the average value of $\frac{1}{2} \operatorname{Tr} U=\phi_{0}^{\pi}$ over space, as a function of the FCC single skyrmion crystal size parameter $L$. Dimensionless units are used for $E / B$ (in units of $6 \pi^{2} f_{\pi} / e$ ) and $L$ (in units of $\left(e f_{\pi}\right)^{-1}$ ). For massless pions, for a value of $L \sim 3.8$ the slope of $E / B$ changes indicating that the system undergoes a first order phase transition. If we look into the actual baryon number distributions (see figures in the small boxes), we see that the system changes from the FCC single skyrmion crystal to a CC half-skyrmion crystal. In the former phase, a well-localized single skyrmion is located at each FCC lattice site where $\phi_{0}^{\pi}=-1$. In the latter phase, one half of the baryon number carried by the single skyrmion is concentrated at each FCC site while the other is concentrated on the links where $\phi_{0}^{\pi}=+1$. Both "half-skyrmions" centered at the points where $\phi_{0}^{\pi}= \pm 1$ can hardly be distinguished and therefore they form a CC crystal. This phase transition can be seen more apparently in the quantity $\left\langle\frac{1}{2} \operatorname{Tr} U\right\rangle$ (see the inset figure). In the literature $[\underline{5}$, the vanishing of $\sigma$ is often interpreted as the restoration of the chiral symmetry. 
If we turn on the pion mass (results shown in the figure by open circles), we see no sudden change in the slope of $E / B$ but at sufficiently high baryon number density skyrmion matter behaves as in an approximate half-skyrmion phase.

The phase to the left of the minimum, referred in our work as "homogeneous", is described by a crystal configuration. The phase to the right of the minimum, which we called "inhomogeneous", because the pressure $P \equiv \partial E / \partial V$ is negative and therefore skyrmion matter is unstable against condensation of the skyrmions into dense lumps leaving large volumes of space empty.

We incorporate the pion fluctuations through the Ansatz

$$
U(\vec{r}, t)=\sqrt{U_{\pi}} U_{M}(\vec{r}) \sqrt{U_{\pi}},
$$

where $U_{M}(\vec{r})$ describes the background baryonic matter and $U_{\pi}=\exp \left(i \tau_{a} \tilde{\pi}_{a} / f_{\pi}\right)$ describes the fluctuating pions. Substitution of this Ansatz into (11) leads to

$$
\begin{aligned}
L_{\pi}^{B \neq 0}= & \frac{1}{2} G_{a b}(\vec{r}) \partial_{\mu} \tilde{\pi}^{a} \partial^{\mu} \tilde{\pi}_{b}+\frac{1}{4} m_{\pi}^{2} \operatorname{Tr}\left(U_{M}\right) \tilde{\pi}_{a} \tilde{\pi}_{a} \\
& -\partial_{\mu} \tilde{\pi}_{a} A_{a}^{\mu}(\vec{r})-\varepsilon_{a b c} \tilde{\pi}_{a} \partial_{\mu} \tilde{\pi}_{b} V_{c}^{\mu}(\vec{r})+\cdots,
\end{aligned}
$$

where we have expanded up to the second order in the fluctuating fields. Eq.(15) describes the dynamics of the pion in the dense medium. $G_{a b}(\vec{r}), V_{\mu}^{a}(\vec{r})$ and $A_{\mu}^{a}(\vec{r})$ are the interaction potentials appearing due to background matter.

As a first approximation let's average the potentials over space. Due to the reflection symmetries of the background matter, $\left\langle V_{\mu}^{a}\right\rangle=\left\langle A_{\mu}^{a}\right\rangle=0,\left\langle G_{a b}\right\rangle=\left\langle 1-\phi_{a}^{\pi} \phi_{b}^{\pi}\right\rangle \equiv Z_{\pi}^{2} \delta_{a b}$ and $\left\langle\phi_{0}^{\pi}\right\rangle \equiv \sigma$. Thus, the Lagrangian becomes

$$
L_{\pi}^{B \neq 0}=\frac{1}{2} Z_{\pi}^{2} \partial_{\mu} \tilde{\pi}^{a} \partial^{\mu} \tilde{\pi}_{a}+\frac{1}{2} m_{\pi}^{2} \sigma \tilde{\pi}_{a} \tilde{\pi}_{a} .
$$

The factor $Z_{\pi}$ in front of the kinetic term can be absorbed into the renormalization of the pion fields as

$$
\tilde{\pi}_{a} \rightarrow \tilde{\pi}^{\prime}=Z_{\pi} \tilde{\pi}_{a}
$$

In terms of this newly defined fields $\tilde{\pi}_{a}^{\prime}$, the Lagrangian can be rewritten as

$$
L_{\pi}^{B \neq 0}=\frac{1}{2} \partial_{\mu} \tilde{\pi}_{a}^{\prime} \partial^{\mu} \tilde{\pi}_{a}^{\prime}+\frac{1}{2} m_{\pi}^{2}\left(\sigma / Z_{\pi}^{2}\right) \tilde{\pi}_{a}^{\prime} \tilde{\pi}_{a}^{\prime}
$$

This implies that the pions effective mass in the medium is given by

$$
m_{\pi}^{*}=m_{\pi} \sqrt{\sigma} / Z_{\pi}
$$

Furthermore, we may reinterpret the wave function renormalization factor $Z_{\pi}$ as the ratio between the in-medium decay constant and the free space one,

$$
f_{\pi}^{*} / f_{\pi}=Z_{\pi}
$$

The results on these in-medium quantities (9) and (10) are presented in Figure 2 as a function of the baryon number density. The scales are strongly dependent on the the parameters $f_{\pi}$ and $e$, e.g. the density units in $\rho$ could change considerably, and therefore the shown numbers should not be taken as definitive. However, the qualitative behavior will remain unchanged. In particular, the dependence on the baryon number density showing approximate scaling with the Skyrme parameter $e$ as $\sim e^{-3}$ is a solid statement.

In Figure 2, we show that at low densities $m_{\pi}^{*} / m_{\pi} \sim 1$, while at higher density the ratio decreases down to zero. The density dependence does not appear linear in $\rho$, since the classical background leads automatically to higher powers in the density dependence. At higher densities, 


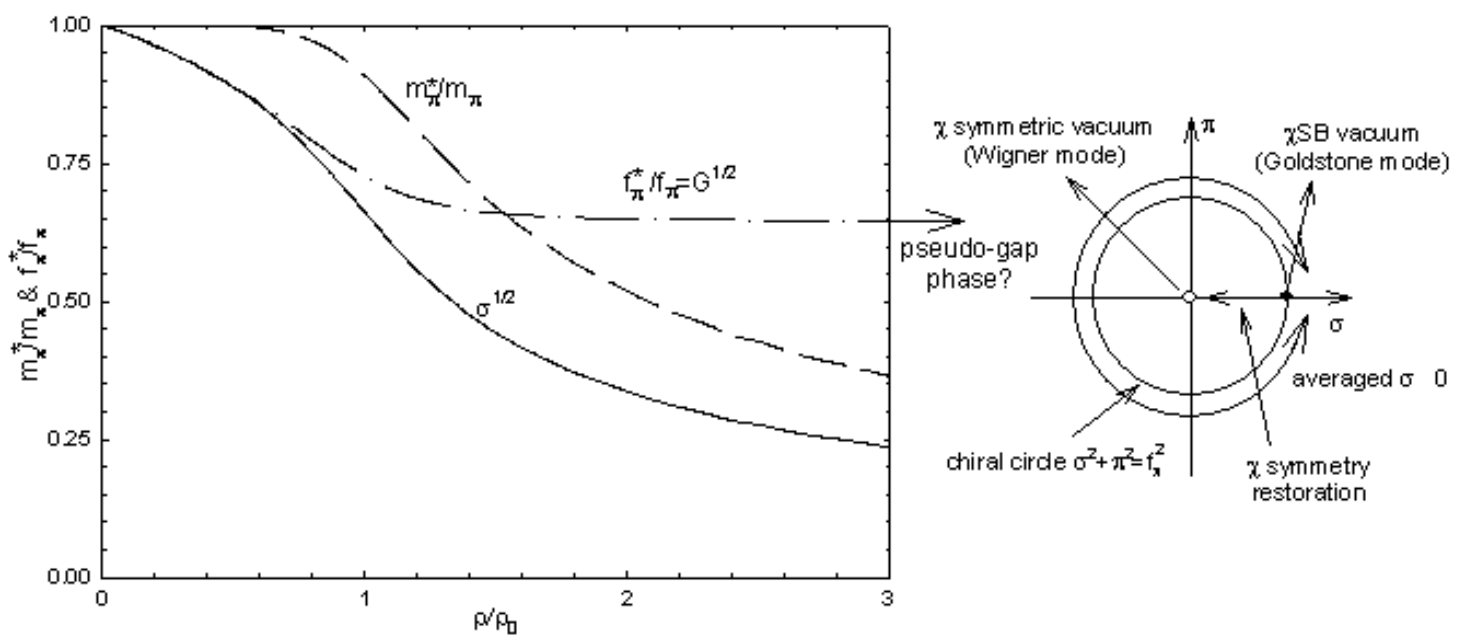

Figure 2: We show the effective pion mass and decay constant in the dense medium as a function of the baryon number density. The dependence on the baryon number density approximately scales with the Skyrme parameter $e$ as $\sim e^{-3}$. The right figure illustrates a pseudo-gap scenario.

powers greater than one in $\rho$ come to play important roles. As the density increases the decay constant $f_{\pi}^{*}$ decreases, which can be interpreted as a partial restoration of the chiral symmetry in the medium. However, the ratio $f_{\pi}^{*} / f_{\pi}$ only decreases up to $\sim 0.65$ remaining constant thereafter. This non vanishing of the pion decay constant, despite the vanishing of $\sigma$, indicates that we may be describing a phase which is not in the standard Wigner-Weyl symmetry. It may be a pseudo-gap phase where the gap is non-zero though chiral symmetry is restored, resembling what might be happening in the normal phase of high $T_{c}$ superconductivity [16]. The pseudo-gap phase is schematically illustrated in Figure 2.

The pseudo-gap phase may be an artifact of the model with only pions. If we have only pion degrees of freedom which are realized non-linearly through the phase of $U$, they must live on the chiral circle and the pseudo-gap phenomenon might appear.

However if we introduce a dilaton field $\chi$, as in (2), we see that it may shrink the chiral circle to a point and the conventional Wigner-Weyl symmetry appears [9]. To illustrate this we take the $\chi$ field constant, $\chi / f_{\chi}=X$. In baryon free space the vacuum of the dilaton field is the minimum point of $V(\chi)$ and is $X=1$. However, for the dense baryonic matter, the ground state should be the minimum of $E / B$, the energy per baryon expressed as

$$
E / B(X)=X^{2}\left(E_{2} / B\right)+\left(E_{4} / B\right)+X^{3}\left(E_{m} / B\right)+\left(2 L^{3}\right) V(X) .
$$

Here, $E_{2}, E_{4}$ and $E_{m}$ are the contributions from the skyrmion field configurations of the given baryon number density through the kinetic term, Skyrme term and mass term of the Skyrme Lagrangian, respectively. Couplings to dilaton matter terms contributes with other effective potentials which will modify the value of $X$ at which $E / B$ has a minimum further.

Shown in Figure 3 is $E / B$ as function of $X$ for various FCC length parameters, where we have substituted for $E_{2} / B, E_{4} / B$ and $E_{m} / B$ the values that led to Figure 1 . At low density (large $L$ ), the minimum of the effective potential $E / B(X)$ is shifted slightly away from $X=1$. As the density increases, $E / B(X)$ starts developing another minimum at $X=0$ which was an unstable extremum in free space. At $L \sim 1 \mathrm{fm}$, the newly developed minimum can compete 


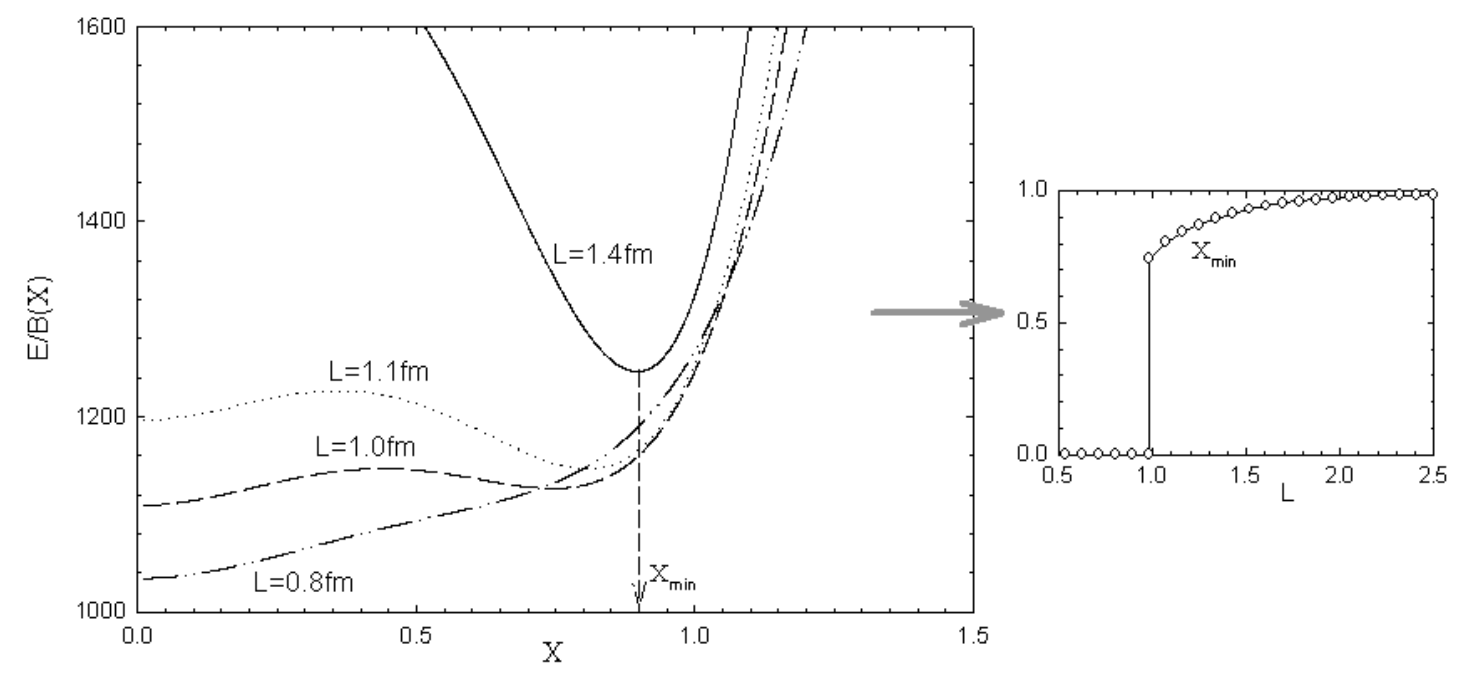

Figure 3: The effective potential $E / B(X)$ as a function of the constant scalar field $X$ for various $L$.

with the one near $X \sim 1$. At higher density, the minimum gets shifted to $X=0$. The figure in the small box presents the resulting values of $X_{\min }$ as a function of $L$. There we see an explicit manifestation of a first order phase transition. This mechanism corresponds to reformulating BR-scaling [15] in a more accurate way.

One can perform a more rigorous treatment by allowing the space dependence in $\chi_{M}(\vec{r})$ (with the symmetries summarized in Table 1), which leads basically to the same physics except for small quantitative difference. The most essential new ingredient is that the static dilaton field for dense matter vanishes identically all over the space in the half-skyrmion phase.

By incorporating the fluctuations of pions and dilaton, as summarized in Table 1, we obtain as before the Lagrangian for the dynamics of these particles

$$
L(U, \chi)=L_{\mathrm{M}, \pi}+L_{\mathrm{M}, \chi}+L_{\mathrm{M}, \pi \chi},
$$

where we have put the subscript " $\mathrm{M}$ " to denote that it describes the in-medium dynamics. Explicitly, each term can be expressed as

$$
\begin{aligned}
L_{\mathrm{M}, \pi}= & \frac{1}{2} G_{a b}(\vec{r}) \partial_{\mu} \tilde{\pi}_{a} \partial^{\mu} \tilde{\pi}_{b}-\frac{1}{2} S(\vec{r}) \tilde{\pi}_{a}^{2} \\
& +\epsilon_{a b c} \partial_{\mu} \tilde{\pi}_{a} \tilde{\pi}_{b} V_{c}^{i}(\vec{r}) \\
L_{\mathrm{M}, \chi}= & \frac{1}{2} \partial_{\mu} \tilde{\chi} \partial^{\mu} \tilde{\chi}-\frac{1}{2} M(\vec{r}) \tilde{\chi}^{2} \\
L_{\mathrm{M}, \chi \pi}= & P_{a}^{i}(\vec{r}) \tilde{\chi} \partial^{i} \tilde{\pi}_{a}+Q_{a}(\vec{r}) \tilde{\chi} \tilde{\pi}^{a} .
\end{aligned}
$$

Here, $G_{a b}(\vec{r}), S(\vec{r}), V_{c}^{i}(\vec{r}), M(\vec{r}), P_{a}^{i}(\vec{r})$ and $Q_{a}(\vec{r})$ are the effective potentials provided to the fluctuating fields by the background fields $U_{M}(\vec{r})$ and $\chi_{M}(\vec{r})$ (For the details, see Ref. [9]).

By applying the same approximation, i.e taking the average values for the effective potentials over the space, we can estimate the medium effects on the properties of the fluctuating fields. As for the pion, we are led to a Lagrangian similar to (6), where $Z_{\pi}$ and $\sigma$ take the average values with the additional factors $\left(\chi_{M} / f_{\chi}\right)^{2}$ and $\left(\chi_{M} / f_{\chi}\right)^{3}$, respectively. Since $\chi_{M}$ vanishes in the chiral symmetry restored phase, the ratio $f_{\pi}^{*} / f_{\pi}$ now vanishes. However, even in the chirally 

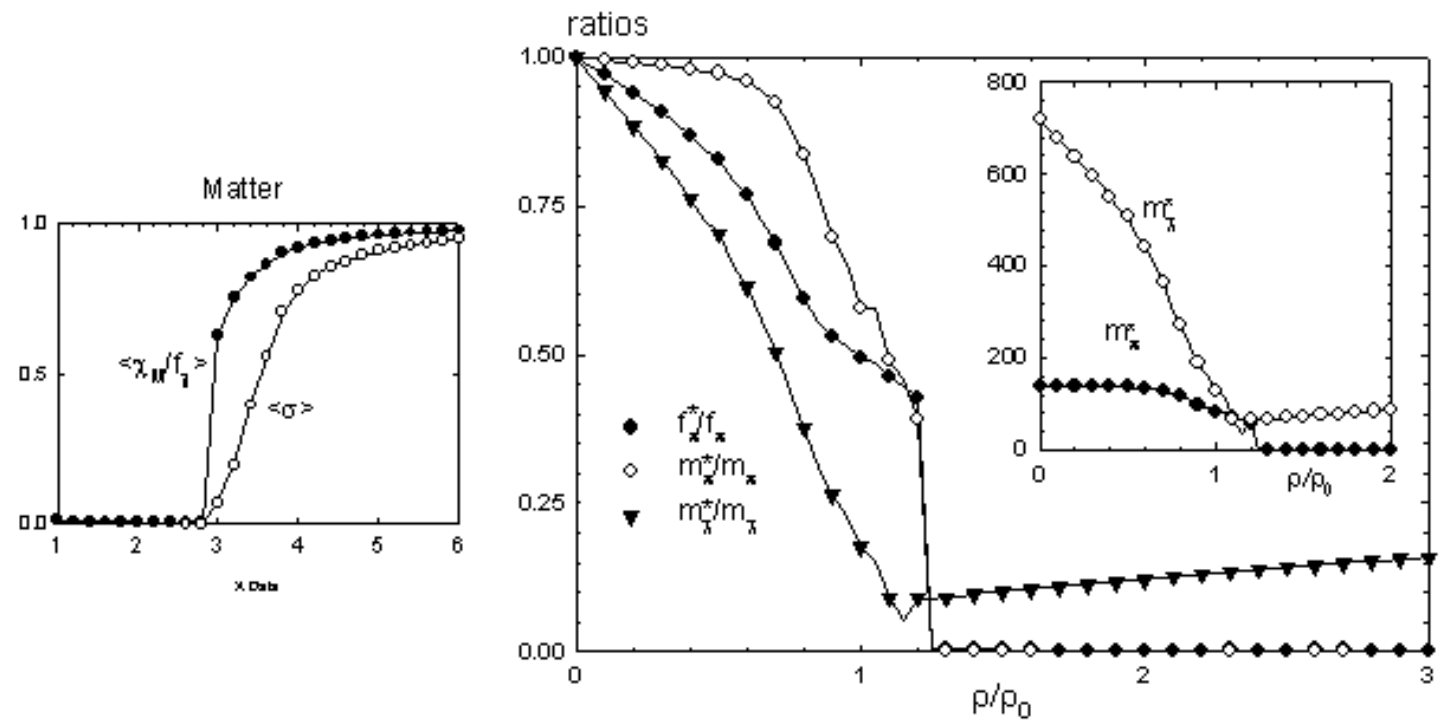

Figure 4: In medium properties of the pion and dilaton fluctuations as a function of the baryon number density. In the inset figure, the pion and dilaton masses are presented in the actual energy scale. The numerical results on the average values of $\chi_{M}$ and $\sigma$ of the background matter are presented in the small figure on the left hand side.

restored phase, with $\langle\sigma\rangle=\left\langle\chi_{M}\right\rangle=0,\langle M\rangle$ does not vanish due to the background $U_{M}(\vec{r})$ couplings to $\tilde{\chi}$. In Figure 4 we show the ratios of the in-medium parameters relative to their free-space values.

The phenomenon discussed above is closely related to "Brown-Rho" scaling [15]. In the description of Ref. [15], the density dependence comes solely from the change in the mean field $\chi^{*}$ where the corresponding change in the skyrmion structure has been ignored. Our present result corrects this fact and gives a precise meaning to the scaling relation of Ref. [15].

We can treat the background interactions more systematically following the perturbative scheme we have developed. We decompose the Lagrangian into an unperturbed part, $L_{0}$, and an interaction part, $L_{I}$. This leads to a Hamiltonian split also in two parts

$$
H=H_{0}+H_{I}
$$

The free propagators are defined by $H_{0}$ and the interaction potentials appearing due to $H_{I}$ are summarized in Figure 5. In Figure 5, $G^{a b}(\vec{\ell})$, for example, is the Fourier transform of the local potential $G^{a b}(\vec{r})$ :

$$
G^{a b}(\vec{\ell})=\frac{1}{V_{\text {box }}} \int_{\text {box }} d^{3} r e^{i \vec{\ell} \cdot \vec{r}} G^{a b}(\vec{r}),
$$

where the integration is over a unit box of the crystal and $V_{\text {box }}$ is its volume. Due to the periodic structure of the crystal only discrete values of the momentum are allowed.

We show in Figure 6 the diagrams used to evaluate the self-energy. Only the diagrams for $\Sigma_{\pi_{a} \pi_{b}}$ appear. The symmetry structure of skyrmion matter allows a non vanishing self-energy only for $a=b$. To first order, $\Sigma^{(1)}$ is nothing but $H_{I}(\vec{\ell}=\overrightarrow{0})$. Since $H_{\pi \chi}(\overrightarrow{0})=0$, no mixing between the fluctuating pions and the fluctuating scalar occurs. Thus, the pion propagator for $\tilde{\pi}_{a}$ can be expressed as

$$
\frac{1}{p_{0}^{2}-\vec{p}^{2}-\Sigma^{(1)}\left(p_{0}, \vec{p}\right)}=\frac{1}{G^{a a}(\overrightarrow{0})\left(p_{0}^{2}-\vec{p}^{2}\right)} .
$$




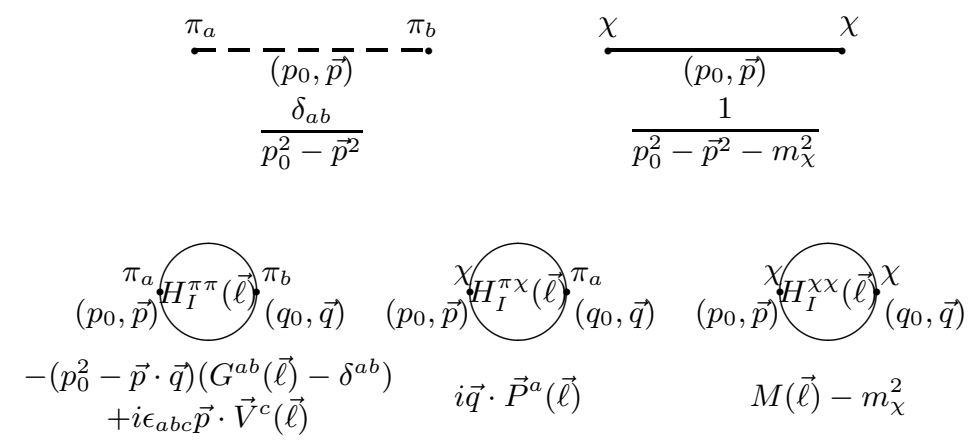

Figure 5: Free propagators and interactions for the pion and the scalar fields in the presence of background skyrmion matter. The energy-momentum conservation delta functions are not shown.

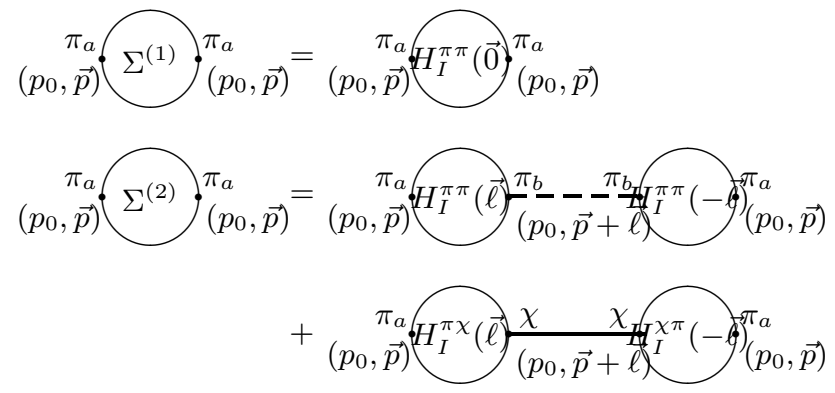

Figure 6: Diagrams used to evaluate the self-energy of the $\pi_{a}$ propagation up to second order in the interaction. Here, $b$ runs over $1,2,3$ and the intermediate states run over all $\vec{\ell} \neq 0$. 
where we have used that the self energy to this order is $\Sigma_{\pi_{a} \pi_{a}}^{(1)}\left(p_{0}, \vec{p}\right)=-p^{2}\left(G_{a a}(\overrightarrow{0})-1\right)$. The superscript "(1)" means that the quantities are evaluated to first order.

Since Lorentz symmetry is broken by the medium, the general form of the in-medium propagator can be written as

$$
\frac{1}{Z_{t}^{-1} p_{0}^{2}-Z_{s}^{-1} \vec{p}^{2}}
$$

with $Z_{t, s}^{-1}=\left(f_{t, s} / f_{\pi}\right)^{2}$ and the "pion velocity" in medium is given as $v_{\pi}^{2}=Z_{t} / Z_{s}$. Comparing with this, we obtain $f_{t}=f_{s}=f_{\pi} \sqrt{G_{a a}(\overrightarrow{0})}$. Since $G_{a a}(\overrightarrow{0})$ is nothing but the average of $G^{a a}(\vec{r})$ over the space, our calculation thus far reproduces the naive approximations discussed above. To the same order, the self-energy of the scalar field is $\Sigma_{\chi \chi}^{(1)}=M(\overrightarrow{0})-m_{\chi}^{2}$. Since it is constant, this self-energy modifies just the scalar mass from the free value $m_{\chi}$ to $\sqrt{M(\overrightarrow{0})}$.

Now, the second order diagrams shown in Figure 6 can be calculated similarly. Again, in spite of the $\pi-\chi$ coupling term in the interaction Lagrangian (12), $\Sigma_{\chi \pi_{a}}^{(2)}$ vanishes so that the pion propagator and the scalar propagator can be simply written as

$$
\frac{1}{p_{0}^{2}-\vec{p}^{2}-\Sigma_{\pi_{b} \pi_{a}}^{(1+2)}}, \quad \frac{1}{p_{0}^{2}-\vec{p}^{2}-m_{\chi}^{2}-\Sigma_{\chi \chi}^{(1+2)}},
$$

respectively.

The $p_{0}$ and $\vec{p}$ dependence of $\Sigma^{(2)}$ is not so simple as that of $\Sigma^{(1)}$. By assuming that energy and momentum are small, we may expand the self-energy $\Sigma_{\pi_{a} \pi_{b}}$ in powers of $p_{0}$ and $\vec{p}$. Then, we can express the propagator in the form of (16), but $Z_{s}$ gets some corrections terms from the second order diagram. The corrections are negative definite so that the pion velocity becomes $v_{\pi}<1$.

The results of our calculation are shown in Figure 7 . We show the decay constants $f_{t}$ and $f_{s}$ in units of $f_{\pi}$ as a function of the density measured in a dimensional units. The inset figure is their ratio, i.e. the in-medium pion velocity $v_{\pi}$. To second order the contribution to $f_{s} / f_{\pi}$ turns out to be small, and thus the pion velocity stays $v_{\pi} \sim 1$. The lowest value is about $\sim 0.9$. Furthermore, for the pions at higher matter densities, the internal propagator provides an extra suppression because $L$ scales as $\rho^{1 / 3}$. Once we pass the density at which the pion velocity has its minimum, the pion velocity increases with density and approaches 1 . When the background matter is in the half-skyrmion phase, $\chi_{0}(\vec{r})$ vanishes identically and so do all the local potentials. Thus, both $f_{t}$ and $f_{s}$ vanish. Moreover, their difference is proportional to the square of the potentials and vanishes faster. This fact explains the asymptotic behavior $v_{\pi} \rightarrow 1$ in Figure 7 . This result is very similar to that of Ref. 17 found in heat bath, where the pion velocity approaches 1 while both the spatial and temporal pion decay constants vanish at $T=T_{c}$.

\section{Vector mesons}

At higher density and/or temperature we need more degrees of freedom such as the $\rho$ and $\omega$ vector mesons. The same procedure can be repeated with the vector mesons using the Lagrangian (3). The lowest energy FCC skyrmion crystal configuration can be found by requiring the symmetries given in Table 1 to the pion, dilaton, rho and omega fields. The numerical results are presented in Figure 8.

In the $\pi \rho \chi$ model, as the density of the system increases ( $L$ decreases), $E / B$ changes slightly. Its value is close to the energy of a single skyrmion up to densities larger than $\rho_{0}(L \sim 1.43)$. 


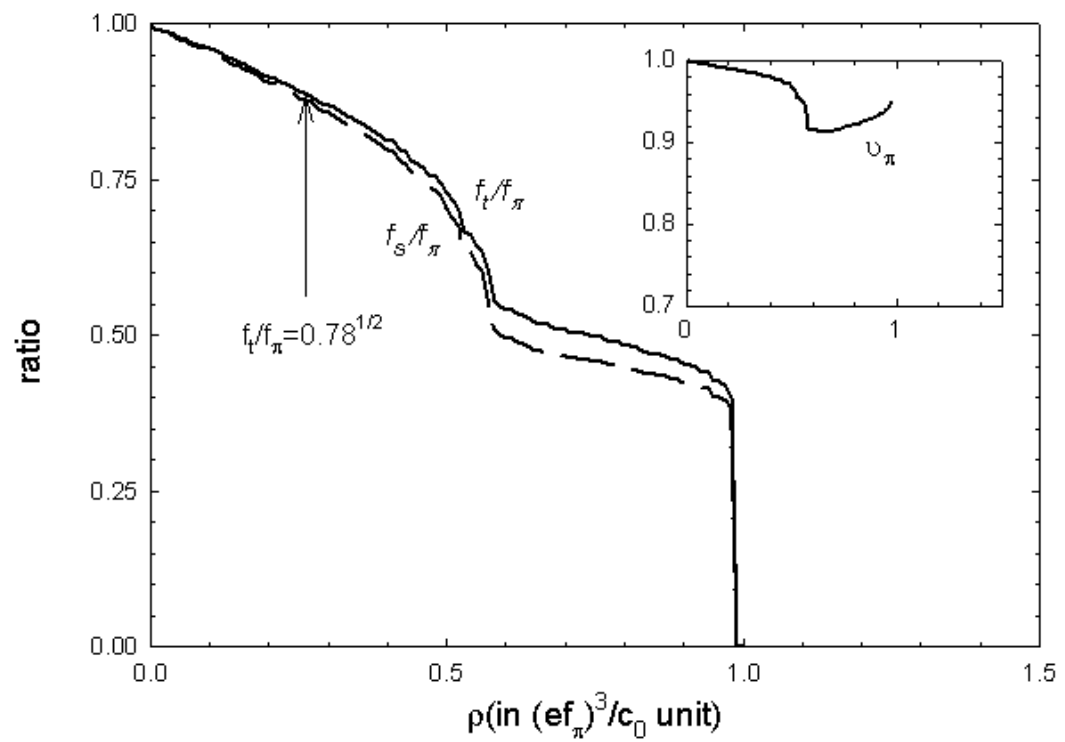

Figure 7: $f_{t}$ and $f_{s}$ as a function of skyrmion matter density in arbitrary units for the chiral limit, $m_{\pi}=0$ and $m_{\chi}=720 \mathrm{MeV}$. The in medium pion velocity appears as function of matter density in the small box. The arrow indicates the point at which the observed ratio in pionic nuclei (at $\rho \approx 0.6 \rho_{0}$ ) is located.

because the size of the skyrmion is very small and the skyrmions in the lattice only interact at very high densities when their tails overlap.

In the absence of the $\omega$ the dilaton field plays as before an important role. Skyrmion matter undergoes an abrupt phase transition at the density at which the expectation value of the dilaton field vanishes $\langle\chi\rangle=0$.

In the $\pi \rho \omega \chi$ model, the situation changes dramatically, because the $\omega$ provides not only a strong repulsion between the skyrmions but also an intermediate range attraction. In both the $\pi \rho \omega$ and the $\pi \rho \omega \chi$ models, at high density, the interaction reduces $E / B$ to $85 \%$ of the $B=1$ skyrmion mass. This value should be compared with $94 \%$ in the $\pi \rho$ model. In the $\pi \rho \chi$-model, $E / B$ goes down to $74 \%$ of the $B=1$ skyrmion mass, but in this case it is due to the dramatic behavior of the dilaton field.

In the $\pi \rho \omega \chi$ model the role of the dilaton field is suppressed. It provides only a small attraction at intermediate densities. Moreover, the phase transition towards its vanishing expectation value, $\langle\chi\rangle=0$, does not take place. Instead, its value grows at high density!

The reason for this can be found in the role played by omega in (3). In the static configuration, omega produces a potential, whose source is the baryon number density, which mediates the self-interaction energy of the baryon number distribution. Thus, unless it is screened properly by the omega mass, the periodic source filling infinite space will lead to an infinite self-energy. To reduce the energy of the system, the effective $\omega$ mass must grow at high density, for which $\chi$ must grow too. Note the factor $\left(\chi / f_{\chi}\right)^{2}$ in the omega mass term in Lagrangian (3). 

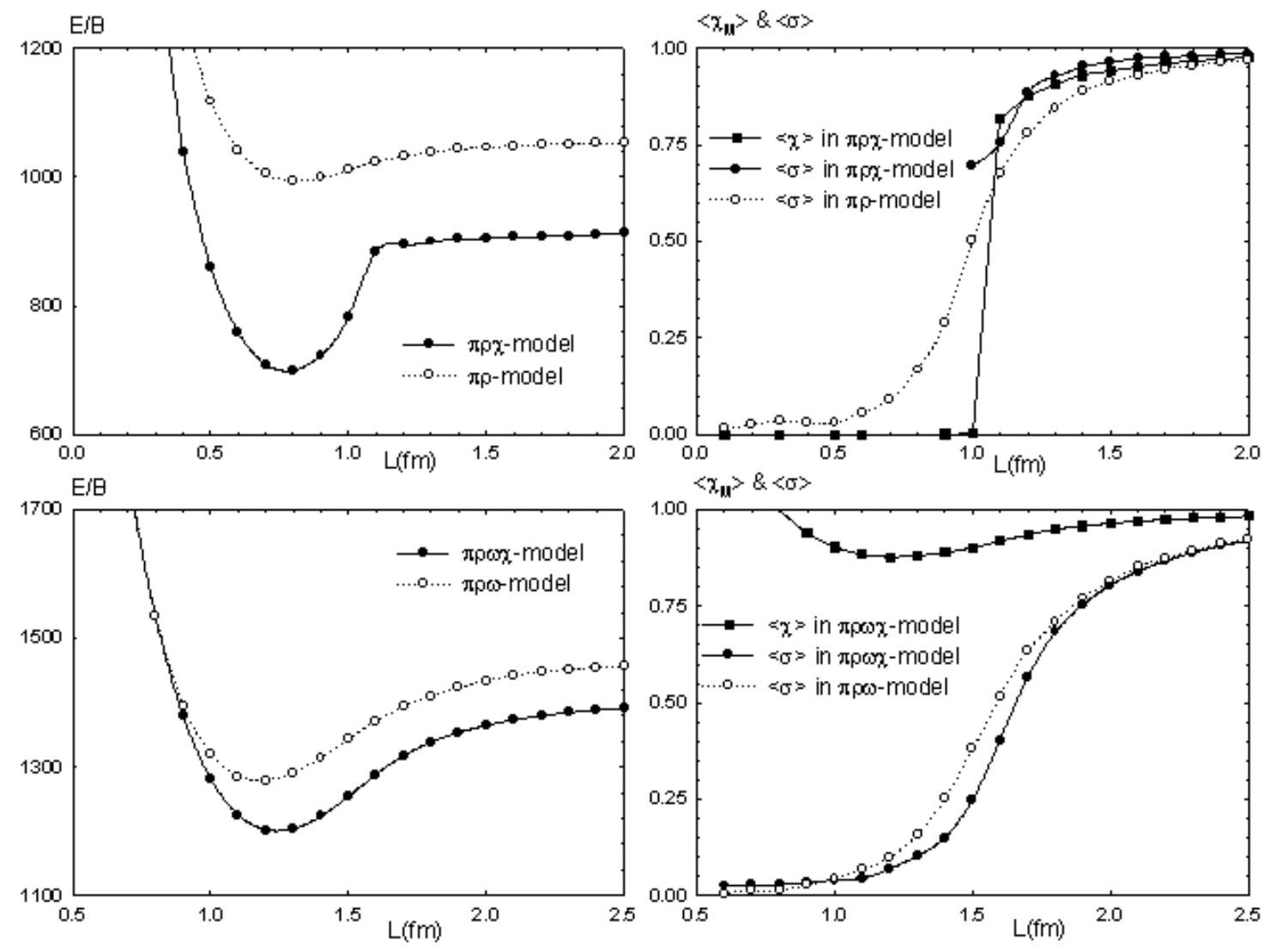

Figure 8: $E / B$ and the average values of the fields over the space as a function of $L$.

\section{Summary}

We have developed a unified approach to dense matter in the Skyrme philosophy, where systems of baryons and mesons can be described by a single Lagrangian. In our approach dense baryonic matter is approximated by skyrmion matter in the lowest energy configuration for a given baryon number density. By incorporating in it fluctuating mesons we can get some insights on meson dynamics in a dense medium. Our approach enables us to study this dynamics beyond the first order in the baryon number density. One can continue to work in this direction by incorporating more degrees of freedom, by improving the way of treating matter beyond the crystal solution, and so on.

However, before closing the presentation, we must clearly lay down the scope of our work. We do not claim that the results obtained at present describe reality. The most fundamental problem we phase is that our "ground state" for matter is a crystal not a Fermi liquid. Our aim has been to assume a state for matter, given by a classical solution of a theory considered to be valid at large $N_{c}$, and have studied the implications for its excitations. Our work should be taken as representing the first step towards a more realistic treatment of a dense matter theory. 


\section{Acknowledgments}

H.-J. Lee, B.-Y. Park and V. Vento are grateful for the hospitality extended to them by KIAS. This work was partially supported by grants MCyT-FIS2004-05616-C02-01 and GVGRUPOS03/094 (VV) and KOSEF Grant R01-1999-000-00017-0 (BYP \& DPM).

\section{References}

[1] For a review, see, for example, T. DeGrand, "Lattice QCD at the end of 2003", hep-ph/0312241; also see T. Hatsuda in this proceedings.

[2] See, for instance, D. K. Hong, in this proceedings.

[3] M. Harada and K. Yamawaki, Phys. Rep. 381, 1 (2003), hep-ph/0302103.

[4] T. H. R. Skyrme, Nucl. Phys. 31, 556 (1962).

[5] I. Klebanov, Nucl. Phys. B262, 133 (1985); G. E. Brown, A. D. Jackson and E. Wüst, Nucl. Phys. A468, 450(1985); A. S. Goldhaber and N. S. Manton, Phys. Lett. B198, 231 (1987); A. D. Jackson and J. J. M. Verbaarschot, Nucl. Phys. A501, 419 (1988); L. Castillejo, P. S. J. Jones, A. D. Jackson, J. J. M. Verbaarschot and A. Jackson, Nucl. Phys. 501, 801 (1989).

[6] M. Kugler and S. Shtrikman, Phys. Lett. B208, 491 (1988); Phys. Rev. D40, 3421(1989).

[7] B.-Y. Park, D.-P. Min, M. Rho and V. Vento, Nucl.Phys. A707 (2002) 381, nucl-th/0201014,

[8] H.-J. Lee, B.-Y. Park, D.-P. Min, M. Rho and V. Vento, Nucl.Phys. A723, 427 (2003), hep-ph/0302019.

[9] H.-J. Lee, B.-Y. Park, M. Rho and V. Vento, Nucl. Phys. A726 69, (2003), hep-ph/0304066.

[10] H.-J. Lee, B.-Y. Park, M. Rho and V. Vento, "Pion Velocity in Dense Skyrmion Matter", hep-ph/0307111, submitted to Phys. Lett. B.

[11] B.-Y. Park, M. Rho and V. Vento, "Vector Mesons and Dence Skyrmion Matter", hep-ph/0310087, submitted to Nucl. Phys. A.

[12] J.-I. Kim, B.-Y. Park, D.-P. Min, M. Rho, V. Vento, in preparation.

[13] J. Ellis and J. Lanik, Phys. Lett. B150, 289 (1985).

[14] M. Bando, T. Kugo, S. Uehara, K. Yamawaki and T. Yanagida, Phys. Rev. Lett. 54, 1215 (1985); Phys. Rep. 164, 217(1988); Ulf-G. Meissner, Phys. Rep. 161, 213 (1988).

[15] G.E. Brown and M. Rho, Phys. Rev. Lett. 66 (1991) 2720.

[16] See e.g. M. Frantz, Z. Tesanovic and O. Vafec, Phys. Rev. B66 054535 (2002), cond-mat/0203333.

[17] M. Harada, Y. Kim, M. Rho and C. Sasaki, "Effective degrees of freedom at chiral restoration and the vector manifestation in HLS theory, Nucl. Phys. A, in press, hep-ph/0308237; M. Harada, Y. Kim, M. Rho and C. Sasaki, Nucl. Phys A727, 437 (2003), hep-ph/0207012. 
Table 1: Summary of the properties of the $B=0$ vacuum solution,the hedgehog Ansatz for the $B=1$ skyrmion, the symmetries of the FCC single skyrmion crystal for pions, dilaton and vector mesons.

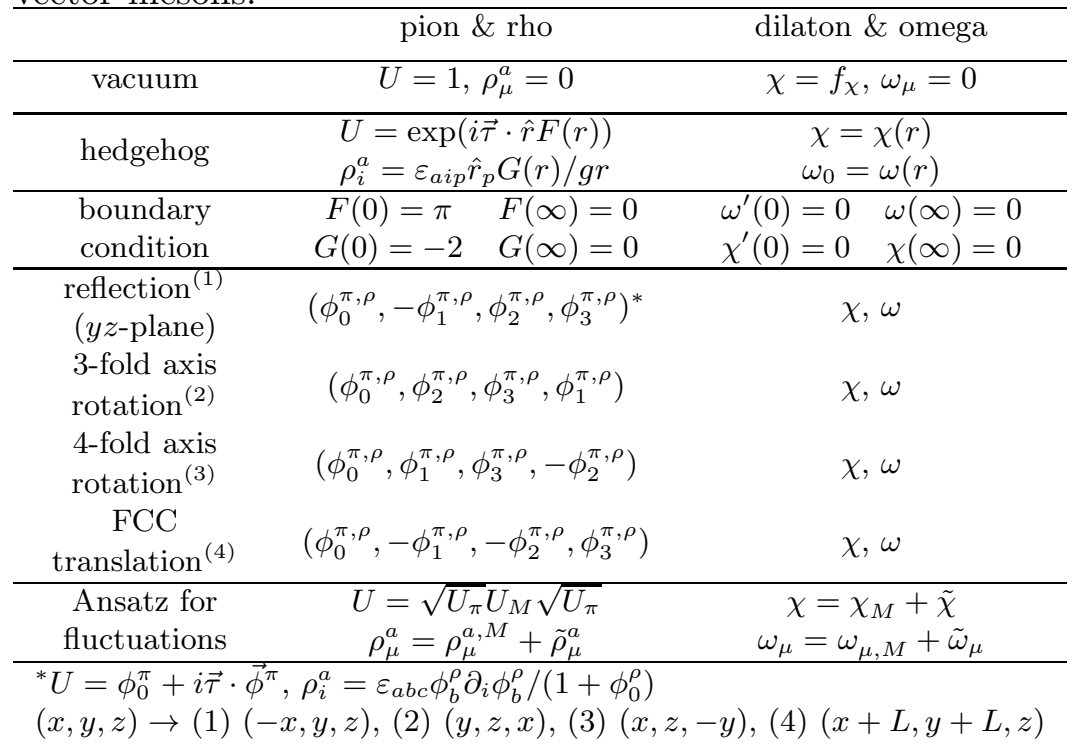

